\title{
Doppler characteristics analysis of forward-looking SAR in helicopters
}

\author{
SUN Yuhui ${ }^{1,2, a}$, WANG Jingen ${ }^{2, b}$, WU Haibing ${ }^{2, c}$
}

1. Department of Weapon Engineering, Navy University of Engineering, Wuhan 430033, China;

2. New Star Research Institute of Applied Technology, Hefei 230031, China;

asunyh389@163.com, bwjg1288@sina.com,2314544434@qq.com

Keywords: Doppler characteristics; forward-looking SAR; bistatic.

\begin{abstract}
As limited by the size and load, multi-array antenna can not be installed in the helicopters to achieve forward-looking SAR imaging. A "bistatic" forward-looking imaging program is proposed, which used a satellite to carry illuminator and a helicopter to carry receiver. Then the forward-looking SAR geometry model and echo signal model are built. At last, the Doppler features are deeply analyzed, especially the variation of the Doppler frequency center, Doppler frequency rate and Doppler frequency third term when depression angle and receiver altitude change. The results provide a theoretical support for forward-looking SAR imaging algorithm and azimuth resolution research.
\end{abstract}

\section{Introduction}

As the beam of airborne forward-looking SAR can be guided autonomous landing of aircraft, in the military, the platform can find target earlier, track and hit the target in real-time. When conventional airborne SAR used for forward observation, its distance and azimuth coupling is serious, and both sides fuzzy image will be generated along track direction, which decreased the ability to distinguish the two-dimensional resolution. If the receiving multi-antenna array can be arranged in the airborne along the vertical track direction, the fuzzy image will be overcome. Although the way is suitable for large aircraft platform to meet the requirements as its adequate wingspan size and load, it does not have the practical feasibility for helicopter platform.

For the actuality of helicopters, this paper proposed "bistatic" forward-looking SAR system. Adopted the bistatic SAR system, radar illuminator and receiver will be placed separately on two different platforms. The transmitter can be placed in a GEO satellite or a LEO satellite, and the receiver in a helicopter platform.

\section{Forward-looking SAR Model}

Various configurations of bistatic SAR with satellite illuminator and airborne receiver have different complexity, so it is necessary to convert the geocentric coordinate system into the ground coordinate system. In order to analyze the model conveniently, a geostationary satellites is suitable. Then the ground coordinate system is established with the origin at the target location. At this point, transceiver platforms are still working in translate variant mode, that the flight speeds or paths of transceiver platforms are different. 


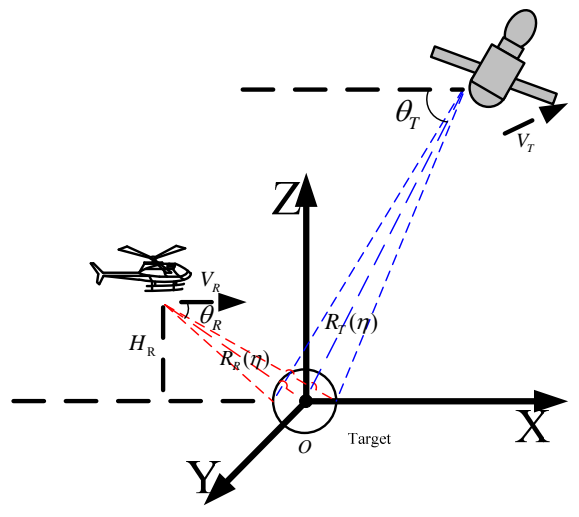

Fig.1 a forward-looking SAR system geometric model

As shown in Fig.1, the receiver platform flights along the $\mathrm{X}$ axis on height, $H_{R}$, at Velocity, $V_{R}$. Transmitter platform is stationary relative to the ground. $O$ is target location, as coordinate origin, $R_{T}(\eta)$ is the distance from illuminator to target, $R_{R}(\eta)$ is the distance from receiver to target. $\theta_{T}$ is the depression angle of the illuminator.

\section{Echo Signal Model}

According to above section, satellite illuminator, which is equipped forward-looking SAR system, transmits chirp signal, and the signal will arrive at the receiver while reflected by the object.

Assuming the chirp signal transmitted is:

$S_{T}(t)=\omega(t) \exp \left(j 2 \pi f_{0} \tau+j \pi K_{r} t^{2}\right)$

Where $t$ is fast time and its reference origin is the pulse center, $\omega(t)$ is pulse envelope that generally is approximate rectangle, $f_{0}$ is carrier wave frequency, and $K_{r}$ is Doppler frequency variant rate. The echo signal reflected by the target is:

$$
S_{R}(t, \eta)=A_{0} \omega(t-R(\eta) / c) \omega_{a}(\eta) \exp \left\{j 2 \pi f_{0}(t-R(\eta) / c)+j \pi K_{r}(t-R(\eta) / c)^{2}\right\}
$$

Where $A_{0}$ is target scattering coefficient, $\eta$ is azimuth slow time, $\omega_{a}(\eta)$ is function of received signal strength with the slow time, $c$ is light velocity, $R(\eta)$ is distance sum of dual transceiver stations to the target point. The received signal, which contains carrier wave, needs to be demodulated orthogonally, and then the baseband signal of single point target can be derived as follows:

$$
S(t, \eta)=A_{0} \omega_{r}(t-R(\eta) / c) \omega_{a}(\eta) \exp \left\{-j 2 \pi f_{0} R(\eta) / c\right\} \exp \left\{j \pi K_{r}(t-R(\eta) / c)^{2}\right\}
$$

This is the general signal recorded and downloaded by SAR systems, also known as raw data. The system processes the signal and obtains the azimuth and range resolution.

\section{Doppler Characteristics Analysis}

The distance of dual transceiver stations to the target is crucial to Doppler characteristic analysis. As mentioned above, the azimuth and range resolution are depend on pulse compression technology. But the azimuth resolution becomes complicated as the variability of Doppler Effect, so it relies on relative motion of the dual transceiver stations to the target.

As mentioned above, the paper analyzes the forward-looking SAR, supposed the transmitter is mounted on a geostationary satellite. And then, the distance from transmitter to the target can be approximated as a constant value, recorded as $R_{\mathrm{TO}}$, so the sum distance is:

$$
R(\eta)=R_{T}(\eta)+R_{R}(\eta)=R_{\mathrm{TO}}+\sqrt{R_{R O}^{2}+V_{R}^{2} \eta^{2}-2 R_{R O} V_{R} \eta \cos \theta_{R}}, \quad-T_{\text {syn }} / 2 \leq \eta \leq T_{\text {syn }} / 2
$$

Where $T_{\text {syn }}$ is synthetic aperture time. Expand formula (4) with Taylor series at $\eta=0$, when the beam center crossing the target. The formula (4) is equal to:

$$
R(\eta)=R_{\mathrm{TO}}+R_{R O}-V_{R} \cos \theta_{R} \eta+\frac{V_{R}^{2} \sin ^{2} \theta_{R} \eta^{2}}{2 R_{R O}}+\frac{V_{R}^{3} \sin ^{2} \theta_{R} \cos \theta_{R} \eta^{3}}{2 R_{R O}^{2}}+\ldots
$$


According to formula (3), the Doppler phase of forward-looking SAR is:

$\varphi(\eta)=-2 \pi f_{0} R(\eta) / c=-2 \pi R(\eta) / \lambda$

Where $\lambda$ is the wavelength of the transmitted signal carrier wave. At this moment, the Doppler frequency of forward-looking SAR is:

$f_{d}(\eta)=\varphi^{\prime}(\eta) / 2 \pi=-R^{\prime}(\eta) / \lambda$

By the formula (5), the formula (7) is equal to:

$f_{d}(\eta)=\frac{V_{R} \cos \theta_{R}}{\lambda}-\frac{V_{R}^{2} \sin ^{2} \theta_{R} \eta}{2 R_{R O} \lambda}-\frac{3 V_{R}^{3} \sin ^{2} \theta_{R} \cos \theta_{R} \eta^{2}}{2 R_{R O}^{2} \lambda}+\ldots$

Therefore, the Doppler frequency center, Doppler frequency variation rate and Doppler frequency third term are:

$$
\begin{aligned}
& f_{d c}=\left.f_{d}(\eta)\right|_{\eta=0}=\frac{V_{R} \cos \theta_{R}}{\lambda} \\
& f_{d r}=\left.f_{d}^{\prime}(\eta)\right|_{\eta=0}=-\frac{V^{2} \sin ^{2} \theta_{R}}{R_{R O} \lambda} \\
& f_{d t}(\eta)=\left.f_{d}^{\prime \prime}(\eta)\right|_{\eta=0}=-\frac{3 V_{R}^{3} \sin ^{2} \theta_{R} \cos \theta_{R}}{R_{R O}{ }^{2} \lambda}
\end{aligned}
$$

\section{Simulation Results and Conclusions}

In order to analyze the Doppler characteristics, all simulation parameters are given as Tab.1.

Tab.1 Parameters of signal transmitted by Radar

\begin{tabular}{cccccc}
\hline Name & Symbol & Value & Name & Symbol & Value \\
\hline wavelength & $\lambda$ & $3 \mathrm{~cm}$ & synthetic aperture time & $T_{\text {syn }}$ & $2 \mathrm{~s}$ \\
signal bandwidth & $\mathrm{B}$ & $20 \mathrm{MH}$ & distance from transmitter to target & $R_{\text {To }}$ & $35860 \mathrm{k}$ \\
pulse width & $T_{p}$ & $5 \mu \mathrm{s}$ & receiver altitude & $H_{R}$ & $2000 \mathrm{~m}$ \\
pulse repetition frequency & PRF & $200 \mathrm{~Hz}$ & receiver flight speed & $V_{R}$ & $80 \mathrm{~m} / \mathrm{s}$ \\
\hline
\end{tabular}

The Doppler frequency of forward-looking SAR is expressed in Fig.2. In this mode, Doppler frequency is a nonlinear function with slow time, and the Doppler frequency bandwidth changes with depression angle. From the four cases illustrated in Fig.2, Doppler frequency bandwidth achieves to the maximal when depression angle is about $55^{\circ}$, is greater than when depression angle is $20^{\circ}, 45^{\circ}$ or $70^{\circ}$. Large bandwidth is conducive to improve the forward-looking SAR azimuth resolution, so it infers that the azimuth resolution will be better when depression angle is about $55^{\circ}$.

In the later imaging process, walking distance correction and geometric distortion correction are all based on the value of the Doppler frequency center, therefore the Doppler frequency center directly affects the quality of post-Doppler imaging quality. Fig.3 illustrates that Doppler frequency center decreases while depression angle increasing. 


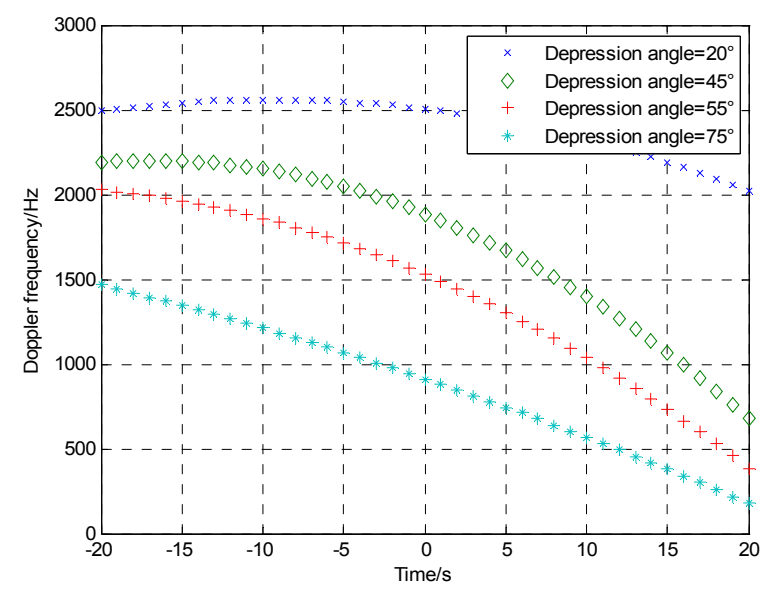

Fig.2 the forward-looking SAR Doppler frequency

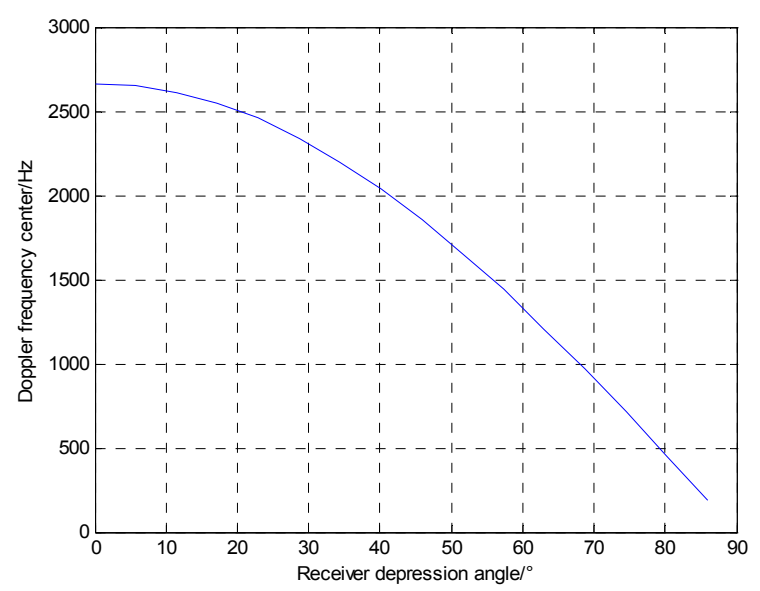

Fig.3 the variety of Doppler frequency center followed with depression angle

Fig. 4 shows that Doppler frequency rate is not a monotone function with depression angle. It obtains the minimum when depression angle is about $55^{\circ}$. As the Doppler frequency rate is always negative, the Doppler frequency declines most sharply when the Doppler frequency rate achieves the minimum (the absolute value is the maximal.)

In this mode, the Doppler frequency is the high order function of slow time. Therefore the impact of Doppler third order term must be considered. Fig.5 is the Doppler frequency third order term followed with depression angle. According to Fig.5, it shows that the Doppler frequency third term obtains the minimum when depression angle is about $40^{\circ}$

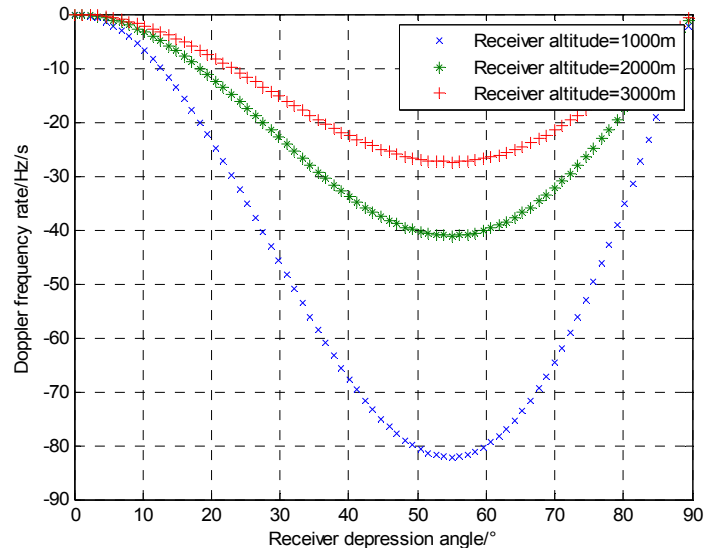

Fig.4 the variety of Doppler frequency followed with depression angle

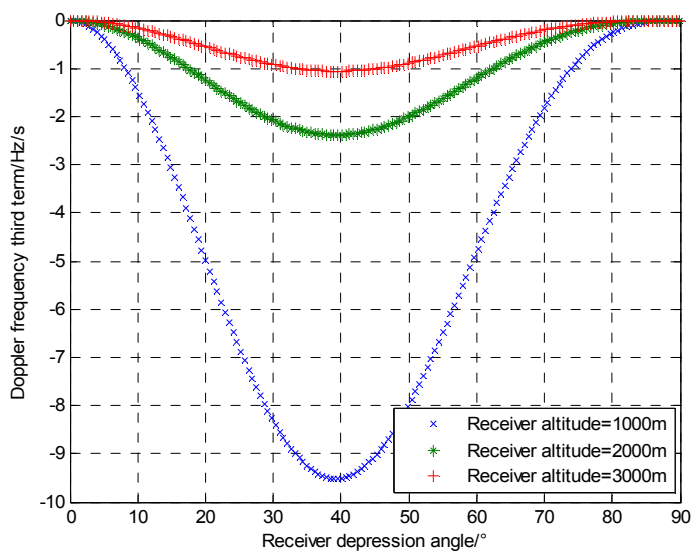

Fig. 5 the variety of Doppler frequency third term followed with depression angle

\section{Conclusions}

According to the above simulation results, it is known that Doppler frequency of forward-looking SAR has its particularity. The azimuth resolution is no longer determined by the antenna synthetic aperture, but is mainly affected by receiver altitude, flying velocity, depression angle, and frequency of carrier wave and so on. The paper presents a new way to analyze the Doppler characteristics, establishes the geometry model and echo signal model. Furthermore, it deduces the detailed expression for each Doppler parameter, and analyses their relationship with the receiver altitude and depression angle by simulation. It provides theoretical support for further research on forward-looking SAR imaging algorithm and azimuth resolution.

\section{References}

[1] Walterscheid I, Espeter T, Klare J, et al. Bistatic spaceborne-airborne forward-looking SAR[C]. Synthetic Aperture Radar 2010 EUSAR, 8th European Conference on. VDE, 2010: 1-4. 
[2] Zhang S S, Li J. Forward-looking bistatic SAR imaging based on high-order range equation and high-order phase compensation[J]. Journal of Electromagnetic Waves and Applications, 2012, 26(17):2304-2314.

[3] Li W, Yang J, Huang Y, et al. A geometry-based Doppler centroid estimator for bistatic forward-looking SAR[J]. Geoscience and Remote Sensing Letters IEEE, 2012, 9(3):388-392.

[4] Wang J, Wang Y, Chen R, et al. Research on the ground resolution of bistatic forward-looking SAR with geostationary illuminator and UAV receiver[C]. Synthetic Aperture Radar, 2012. EUSAR. 9th European Conference on. VDE, 2012:567-570.

[5] Li W, Yang J, Huang Y, et al. An improved radon-transform-based scheme of Doppler centroid estimation for bistatic forward-looking SAR[J]. Geoscience and Remote Sensing Letters IEEE, 2011, 8(2): 379-383.

[6] Hou Hai-ping, Qu Chang-wen, Ding Can et al. Influence of Array Antenna Micro-motion on Forward-looking SAR Imaging and Compensation Study [J]. Journal of Electronics \& Information Technology, 2011, 33(4):831-837.

[7] $\mathrm{J} \mathrm{Wu}, \mathrm{Z} \mathrm{Li}, \mathrm{Y}$ Huang, et al. Focusing Bistatic Forward-Looking SAR With Stationary Transmitter Based on Keystone Transform and Nonlinear Chirp Scaling[J]. IEEE Geoscience \& Remote Sensing Letters, 2014, 11(1):148-152.

[8] Wang X, Zhu D, Zhu Z. Research on the bistatic forward-looking sar imaging[J]. Journal of Electronics, 2010, 27(06):735-741.

[9] Franceschetti G, Iodice A, Riccio D. Forward-Looking Synthetic Aperture Radar (FLoSAR): The Array Approach[J]. IEEE Geoscience \& Remote Sensing Letters, 2014, 11(1):303-307.

[10]Qi C D, Shi X M, Bian M M, et al. Focusing forward-looking bistatic SAR data with chirp scaling[J]. Electronics Letters, 2014, 50(3):206-207. 